\title{
A NOTE ON THE WEIGHTED \\ HARMONIC-GEOMETRIC-ARITHMETIC MEANS INEQUALITIES
}

\section{GÉRARD MAZE AND URS WAGNER}

Abstract. In this note, we derive non trivial sharp bounds related to the weighted harmonicgeometric-arithmetic means inequalities, when two out of the three terms are known. As application, we give an explicit bound for the trace of the inverse of a symmetric positive definite matrix and an inequality related to the coefficients of polynomials with positive roots.

Mathematics subject classification (2010): Primary 26D15; Secondary 15A42.

Keywords and phrases: Classical means, weighted HGA inequalities, sharp inequalities.

\section{REFERENCES}

[1] Z. BAI AND G. H. Golub, Bounds for the trace of the inverse and the determinant of symmetric positive definite matrices, Ann. Numer. Math., 4 (1997), 29-38.

[2] E. F. Beckenbach And R. Bellman, Inequalities, Second revised printing, Ergebnisse der Mathematik und ihrer Grenzgebiete, Neue Folge, Band 30 Springer-Verlag, New York, Inc. 1965.

[3] A. Fransén and J. Lohne, Problem 196, Nad. Mat. Tidskr., 8 (1960), 188.

[4] G. H. Hardy, J. E. Littlewood, And G. Polya, Inequalities, 2d ed. Cambridge, University Press, 1952.

[5] G. V. Milovanović, D. S. Mitrinović, And Th. M. Rassias, Topics in polynomials: extremal problems, inequalities, zeros, World Scientific Publishing Co. Inc., River Edge, NJ, 1994.

[6] D. S. Mitrinović, Analytic inequalities, In cooperation with P. M. Vasić, Die Grundlehren der mathematischen Wissenschaften, Band 165 Springer-Verlag, New York-Berlin, 1970.

[7] Z. PÁles, Essential inequalities for means, Periodica Math. Hungar., 21, 1 (1990), 9-16. 